\title{
FAST METEOROLOGICAL FORECASTING METHOD FOR CHEMICAL SPILL IN BLACK SEA
}

\author{
Dinu ATODIRESEI, Ionuț-Cristian SCURTU, \\ Florin NICOLAE, Petru Sergiu ȘERBAN \\ "Mircea cel Bătrân" Naval Academy, Constanța, Romania \\ dinu.atodiresei@anmb.ro
}

\begin{abstract}
The Romanian Navy missions and actions in Black Sea can lead to ecological risks and the forecast of spill direction based on real data from NOOA. There is an increase in strategic importance of the Black Sea region and the protection of Romanian coastline is a very important task. Chemical spill can now be analyzed with a fast method, called Gnome and predictions can be used with higher accuracy. Forecasts can be used in port protection against offshore spill and as military source of high value information related to Black Sea.
\end{abstract}

Keywords: forecast, chemical, Gnome, Black Sea.

\section{Introduction}

Based on Navy missions and the needs of controlling accidental spill [1] we present a method for forecasting these spills based on software from NOOA. GNOME is a program for modeling the trajectory of oil spills that simulates their movement under the action of wind, currents, tide, calculating the values of time dispersion and surface. We will use this model during actions of limiting discharges to calculate the best prediction of hydrocarbons path.

There is a software package developed by NOAA (National Oceanic and Atmospheric Administration) for responding to cases of oil pollution, which includes the following main programs:

CATS - modeling/ estimating sea currents in well-defined geographical structure to shore and depths defined by position (latitude, longitude) and possible value $(\mathrm{m})$.

GNOME - effective analysis of scattering spilled hydrocarbons problems in the marine environment, providing the use while traveling more terms (Mover) by simply composing current fields; the field of trajectories given by compound can be added direct with effects of wind from the issue of hydrocarbons.

Reactivity of more types of substances spilled allows the risk estimation of dangerous reactions in case of mixed products.

GNOME can be used to:

$>$ forecast wind, currents and other hydro-meteorological factors that can move and disperse hydrocarbons into the water;

$>$ learn how hydrocarbons
trajectory estimates are affected by
inaccurate observations on hydro-
meteorological data of currents, wind and
pollutant nature;
and physically changes during their
movement through the use of
complementary programs ADIOS type-2,


thus achieving an estimated overall budget of remaining quantities of oil spilled after surgery decontamination means [2].

To use GNOME there must be described a scenario spill by entering information into the program using GOODS (GNOME Online Oceanographic Data Server). Gnome then creates and runs a film showing the predicted trajectory of oil discharge from the script.

GNOME can estimate discharge path, processing the information we introduce about wind, weather, currents and discharge that we want to simulate.

Gnome models were created in three ways:

Standard mode;

GIS module;

$>$ Diagnostic mode.

In standard mode a file location data are processed, such as area map and the current dominant models in Gnome. Then ask for the information system needs to run the model. In standard mode you can save the settings for the model, can print photos or create a movie of how to run the model.

Standard module is flexible enough to respond to the spill, but is an excellent tool to simulate discharges and build an intuition about the spill trajectory for specific locations.

The GIS module also uses a file location to set the pattern. In this way the model can be exported in a format GIS compatible (GIS - Geographic Information System)

In diagnosis mode, you can use a file location to set a model or you can set a model by entering other data. Without a file location (Base Maps - Global custom map generator), Gnome requires the introduction of shoreline and hydrodynamic information. In diagnostic mode we have the following additional options:

$>$ full access to model parameters and options scale;

$>$ build the model so that we have real-time data in real time - hydrometeorological data and forecasting;
$>$ with advanced training and experience in hydrodynamic modeling can create a file location;

$>$ set coefficients that control the size and distribution of estimation uncertainty used in 'Minimum Regret'.

Trajectory Simulation (surface currents) flow model is defined in four ways/ distinct approaches:

shaping power lines - SAC (allowing defining contours and amplitude on them);

$>$ modeling of wind currents WAC (the amplitude values can be defined by contours elevation and the level in combination with wind power factor generated on the surface of the sea);

$>$ modeling tidal currents generated by Shut - facility still inoperable in the Black Sea;

$>$ diagnostic model type - DAC (on high dynamic flow conditions that include as parameters: friction depth (linear or not), advection, Coriolis force, highatmosphere coupling coefficient).

\section{starting point}

Modeling power lines - SAC. The

The equation - the current transport function:

$$
\frac{\partial h}{\partial t}+\nabla \bullet(h \vec{V})+\nabla \bullet(d \vec{V})=0
$$

change the flow equation.

Boundary conditions:

$>$ all without limits flow lines are current;

$>$ the difference between the current lines give sewers transport;

$>$ natural flow limits do not prevent flow;

size of change flow is used to relax the constraints of non - divergent and simulate a steady stream of current.

The diagnosis type - $\mathrm{DAC}$

The equation for surface height

$$
\varepsilon \nabla^{2} \xi+J(d, \xi)=0 .
$$

Boundary conditions:

$>$ specific surface height along isobaric have at least an open edge of the 
domain model;

$>$ correct edge surface height is right on the shallow water if we are in the Northern Hemisphere;

$>$ edges of the shoreline may be 'non-flowing' raised levels, less inclined levels depend on the current of the simulated coast; natural limit does not prevent the flow.

Modeling of wind currents - WAC

The equations for surface elevation model

$$
\begin{gathered}
\frac{\partial h}{\partial t}+\nabla \bullet(h \vec{V})+\nabla \bullet(d \vec{V})=0 \\
-f U=-g \frac{\partial \xi}{\partial y}+\frac{\tau_{y}}{\rho(h+\xi)}-\frac{c\left|U^{2}+V^{2}\right|^{\frac{1}{2}}}{(h+\xi)} V
\end{gathered}
$$

Boundary conditions for surface height:

$$
\begin{gathered}
\frac{\partial}{\partial x}[(h+\xi) U]+\frac{\partial}{\partial y}[(h+\xi) V]=0 \\
f V=-g \frac{\partial \xi}{\partial x}+\frac{\tau_{x}}{\rho(h+\xi)}-\frac{c\left|U^{2}+V^{2}\right|^{\frac{1}{2}}}{(h+\xi)} U
\end{gathered}
$$

\section{Method using and following the procedure work}

This section will guide us step by step, in a Gnome scenario describing the installation of the program and the study of an oil spill in the Black Sea.

GNOME is available online: response.restoration.noaa.gov/oil-andchemical-spills/oil-spills/responsetools/downloading-installing-and-runninggnome.html [6].

Gnome will led you through questions immediately (primarily on license - click "OK"), then choose a file location. NOAA type localization files are created in several regions and the data they contain (topography, climatology and tide for that location).

From the File menu, select "Preferences" and then option mode (Fig. 1). Now you have two modes - Standard and Diagnostic.

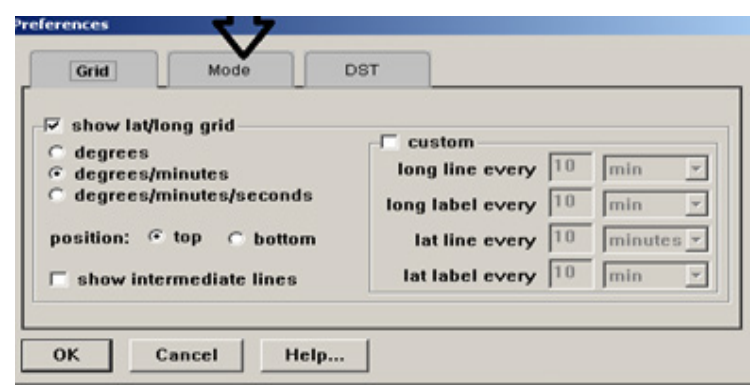

Fig. 1. Selecting the option

Set parameters describing the wind and tide. You can choose default settings or enter your own data measured precisely from the National Institute of Meteorology (NIMH), Maritime Hydrographic Directorate (DHM) or extracts real-time synoptic maps relating to that area (Fig. 2).

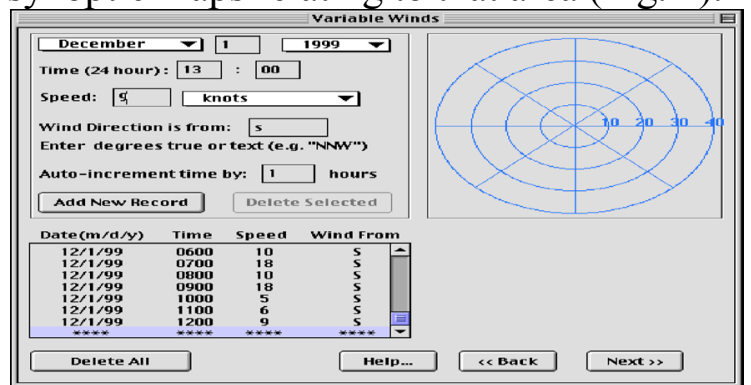

Fig. 2. The settings for the chosen parameters related to the type of wind and wave direction ( 2 cases).

Now we have a map with a previous current, wind and wave selected by us. Choose the selected map coordinates of the point where it was discharged hydrocarbon, call the film (the program allows simultaneous modeling of several discharges), pollutant type, quantity and time of discharge (Fig. 3).

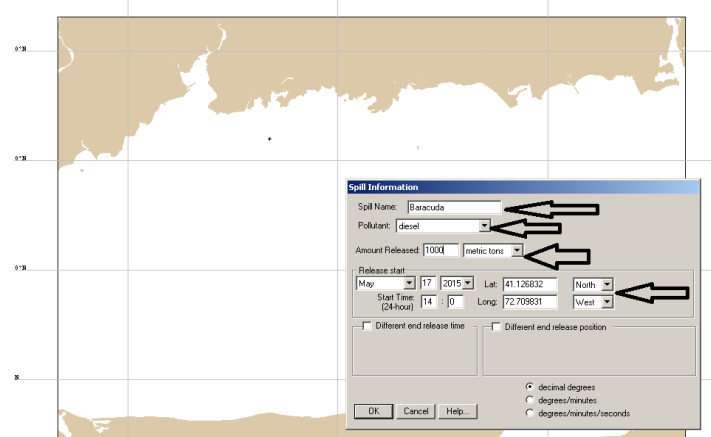

Fig. 3. Entering data for hydrocarbon film

You will notice the process of dispersion modeling of the selected 
hydrocarbon film within the time required and the cursor can select the area held discharge, surface affected and can stop running modeling when hydrocarbon reaches shore.

\section{DIAGNOSTIC mode operation on GNOME}

If you choose Diagnostic mode, the situation becomes "real time" and can thus create:

$>$ Map of the position you are in real time at any point of the globe;

$>$ Hydro-meteorological

information are provided by satellites with parameters forecasting wind and currents over a period of 2 weeks (not recommended due to file size and real time data download speed on the NOOA site;

$>$ Surface Dispersion estimate the amount of discharged hydrocarbons, forecasting meteorological parameters; it will be an input for ADIOS2 program.

\section{following steps:}

Modeling algorithm involves the

$>$ Go to

http://gnome.orr.noaa.gov/ [6]

$>$ Global enter the custom map generator and we will select the area you want to do an analysis of variance hydrocarbons (in our case study, the Black Sea area W) (Fig 4)

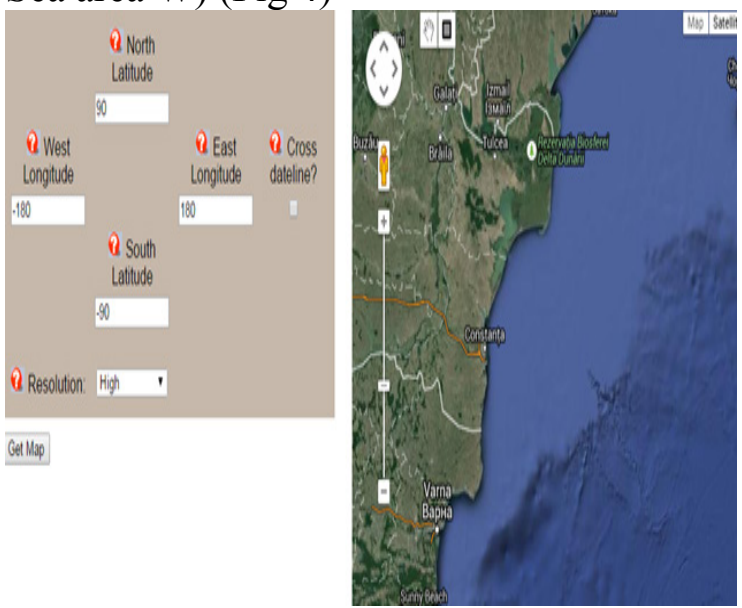

Fig 12 Selecting the area (corresponding map) reference to how Diagnosis

Draw rectangle and press the button we select reference Strip area that interests us for creating paper.

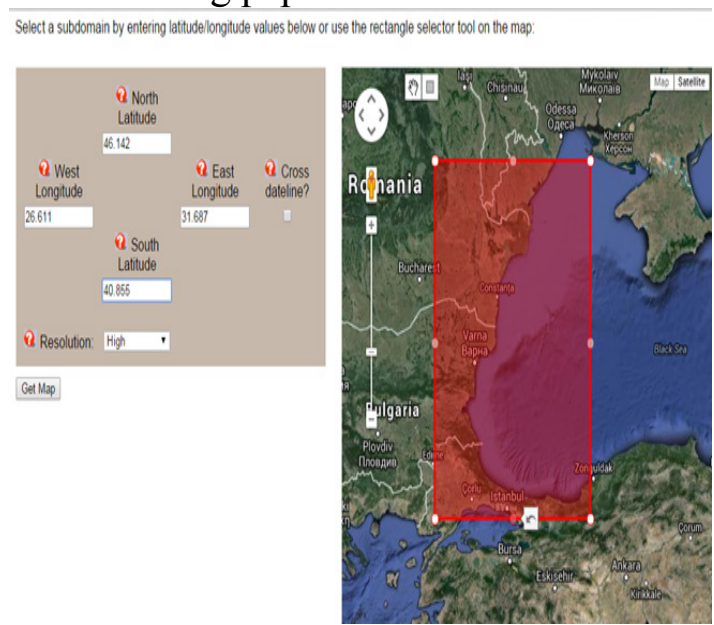

Fig. 4 Collecting paper to a desired level (related to the reference area) for Diagnostic mode

We select button will save get map and that map coast.bna that we directly copy the program for GNOME to dusk then directly map into the program.

After creating the map, the next step is the creation of wind and current files while opening a new page again we get back site http: //gnome.orr noaa.gov / goods and will enter the module Winds (NCEP Global Forecast System) as shown in Fig. 5.

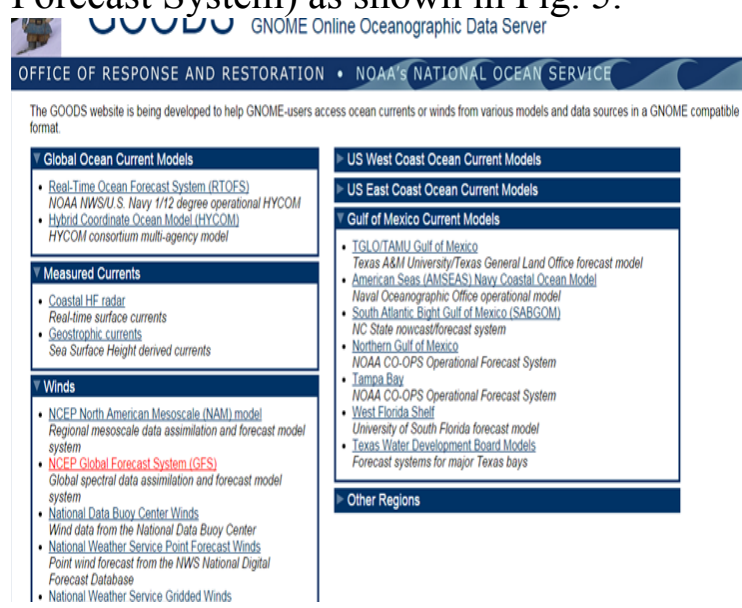

Fig 5 Winds Access Module

We will select the first option and we will submit.

Select last 2 weeks + forecast and we will again submit.

Wind generation data file for the selected map.

As you can see, it was again generated a map (Very important: here we 
copy the coordinates from the first map created new map generator) and for us as pollution discharge date - I took the case study $05 / 29 / 2015$ until $06 / 06 / 2015$ and the dimensions we select update to generate the same map as the one we originally created . We select get time to generate the file with the wind for the area.

Save the file

9GFS_Global_onedeg.nc in the program folder and included in GNOME option movers.

After you select a file get GFS_Global_onedeg.nc will save time as well as if the folderol map created GNOME program. From the program, select movers, continue with the selection variable winds we press load entering the file above GFS_Global_onedeg.nc.

Uploading wind map and display parameters on the selected time interval of its movement.

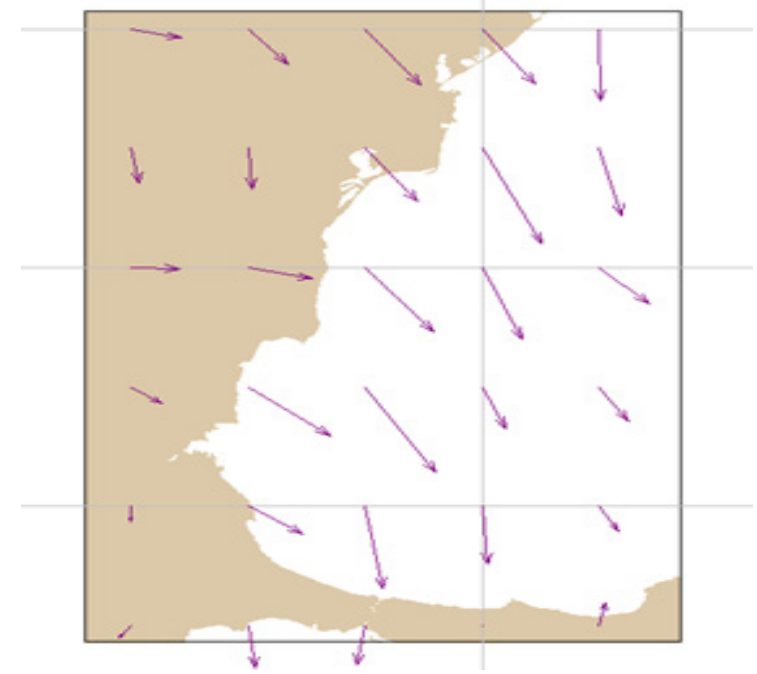

Fig 6 The wind map in real time dispersion analysis

We load the file and select window will appear, select Show velocity and the period of 72 hours (Fig. 6). For current, update and dimensions we select get time, as shown in fig.7.

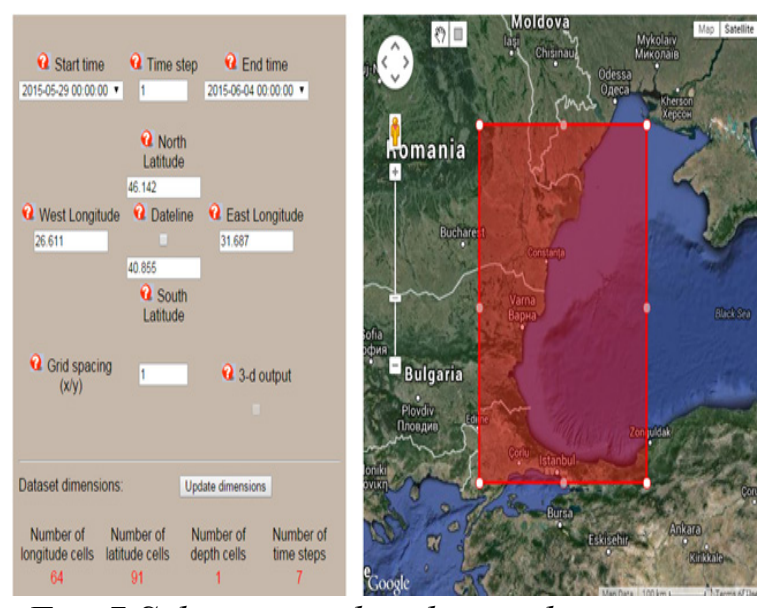

Fig. 7 Selecting and updating the map size in order to create the file type HYCOM.nc

Display on the map of current and related parameters selected time interval of its movement.

\section{Results}

We select load the file and will appear for selection of parameters (Fig. 8,9 ), select show velocity (for the movement) and period (time) 72 hours, press $\mathrm{OK}$ to view the combined action of wind and currents

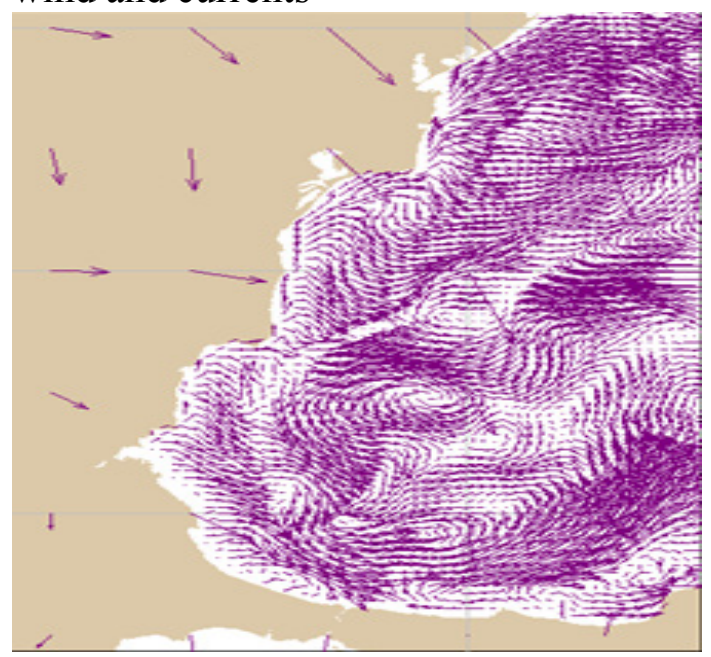

Fig. 8 Capture representation combined action of wind and currents in the area (map) created 


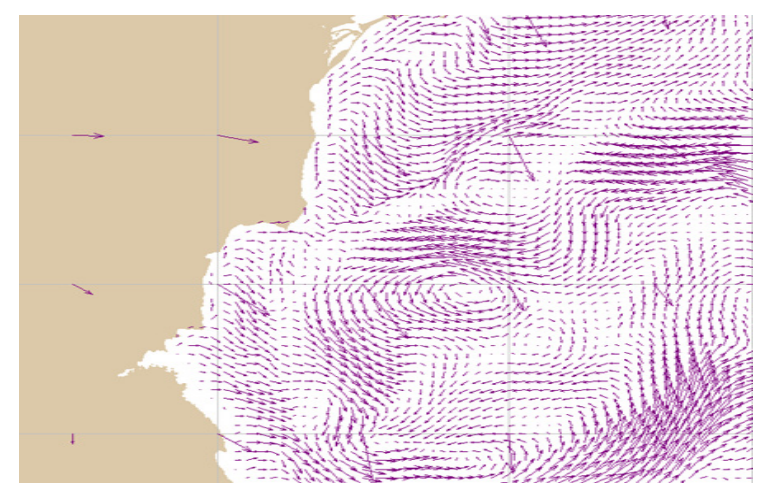

Fig 9 Generating map with real-time wind and current on a larger scale

The next step is to represent the amount of oil spilled (in terms of a default scenario or any real situation in terms of discharge from the board).

Each spills can we ascribe a name different types of hydrocarbon quantities with different physical and chemical properties - the transport of oil as cargo, fuel, etc. (Fig. 10)

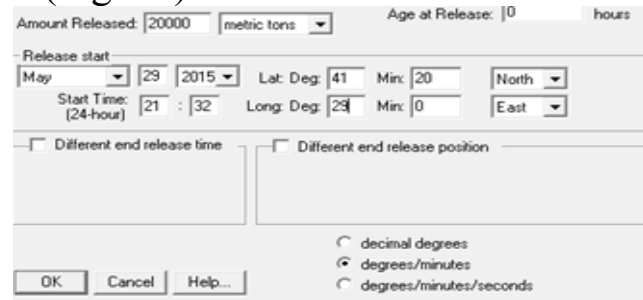

Fig 10 Selecting quantity and position with geographical coordinates.

In fig 11, it can be seen from the map scale (red square) created the film of hydrocarbons.

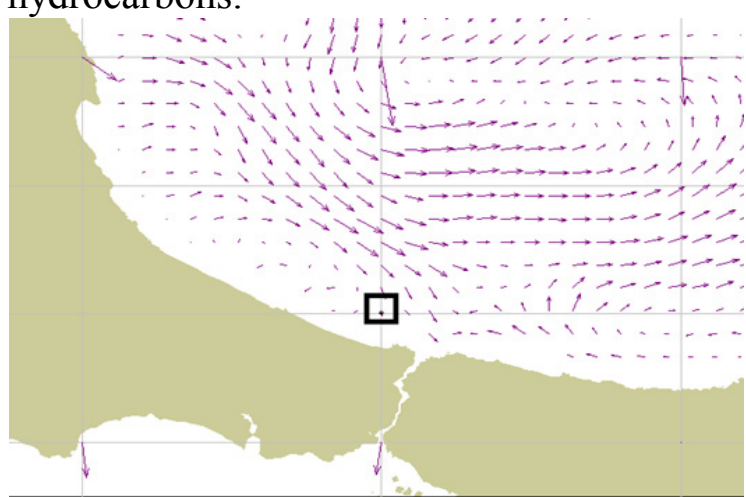

Fig 11 Viewing hydrocarbon film created

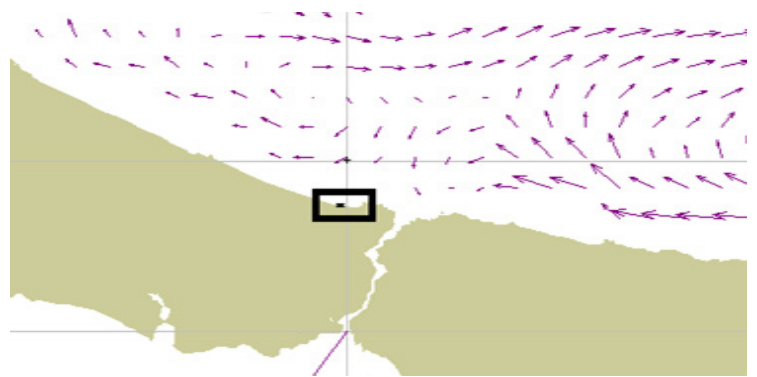

Fig 12:Evolution film hydrocarbon close to coastline

Simulating evolution film hydrocarbons determining the time of arrival on the coast under the influence of meteorological factors, according to forecasts estimated in real time. [4] The scrollbar will present data according to chronology oil dispersion film (Fig 12), on the 31.02.2016 at 13:32 fractions / components in the suspension of hydrocarbon film will reach land.

\section{Conclusion}

The method presented is a powerful tool for understanding risks in case of CBRN predictions and chemical spill. The importance of the forecast of spill direction based on real data from NOOA can lead to a better decision in risk management associated to navy operations in Black Sea [1] [3].

The protection of Romanian coastline is a very important task [4] and results presented from method and tools available in Gnome software.

Software predictions can help navy experts to protect Romanian coastline and in case of war to know where the chemical spill will be driven by wind and currents according to forecasts for 24 or 72 hours.

Forecasts can be used as military source of CBRN high value information related to Black Sea and for offshore marine energy in the Black Sea area [5]. 


\section{References}

[1] Lupu S., Pocora A., Toma A., Katona C., Training of the romanian navy personnel for underway replenishment operations Proceedings of the 11th International Scientific Conference eLearning and Software for Education "Rethinking education by leveraging the eLearning pillarof the digital Agenda for Europe", April 23 - 24, Bucharest, 2015.

[2] Atodiresei D., Clinci C., Dia D., Cretu G., Andriu C., Designing a model based on existing simulators this time inuse by the romanian navy to ensure effective interventions in limiting the effects of military, "Mircea cel Batran" Naval Academy Scientific Bulletin, Volume XIV - 2011 - Issue 1 Published by "Mircea cel Batran" Naval Academy Press, Constanta, Romania

[3] Scurtu I.-C., Minimum Deck Height Of A SemiSubmersible Platform According To Black Sea Environment, Buletinul Stiintific Academiei "Mircea cel Batran" Constanta, Sectiunea Nautical and management science, 2014 - Issue no. 1 Cod CNCS 884, ISSN 2392-8956, ISSN-L 1454-864X, pg.13-18.

[4] Boșneagu R., Scurtu I. C, Weather and oceanographic influence on the maritime navigation, Constanta Maritime University Annals, Year XIV, Vol.21, ISSN 1582-3601, Constanta 2014.

[5] STAN L.-C., Offshore marine energy in the european area, Constanta Maritime University Annals, Year XIV, Vol.24, ISSN 1582-3601, Constanta 2015.

[6] http://response.restoration.noaa.gov/gnome 